\title{
A New Version of Schauder and Petryshyn Type Fixed Point Theorems in S-Modular Function Spaces
}

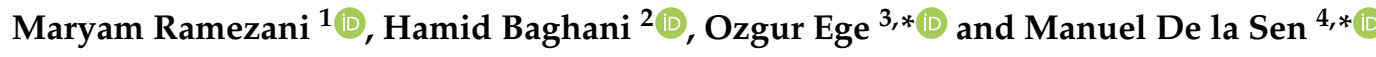 \\ 1 Department of Mathematics, University of Bojnord, 94531 Bojnord, Iran; m.ramezani@ub.ac.ir, \\ mar.ram.math@gmail.com \\ 2 Department of Mathematics, University of Sistan and Baluchestan, Zahedan P.O. box 98155-987, Iran; \\ h.baghani@gmail.com \\ 3 Department of Mathematics, Ege University, Bornova, 35100 Izmir, Turkey; ozgur.ege@ege.edu.tr \\ 4 Institute of Research and Development of Processes University of the Basque Country, \\ 48940 Leioa, Bizkaia, Spain \\ * Correspondence: manuel.delasen@ehu.eus (M.D.1.S.); ozgur.ege@ege.edu.tr (O.E.)
}

Received: 2 December 2019; Accepted: 10 December 2019; Published: 19 December 2019

\begin{abstract}
In this paper, using the conditions of Taleb-Hanebaly's theorem in a modular space where the modular is s-convex and symmetric with respect to the ordinate axis, we prove a new generalized modular version of the Schauder and Petryshyn fixed point theorems for nonexpansive mappings in $s$-convex sets. Our results can be applied to a nonlinear integral equation in Musielak-Orlicz space $L^{p}$ where $0<p \leq 1$ and $0<s \leq p$.
\end{abstract}

Keywords: s-convex set; s-modular function space; fixed point; Musielak-Orlicz space

MSC: 47H10; 54H25

\section{Introduction}

In 1950, Nakano [1] initiated the concept of modular spaces which are natural generalizations of $L^{p}$ spaces where $p>0$. Then Musielak and Orlicz [2] refined and generalized these spaces in 1959 . This idea has been studied for almost sixty years and there is a large set of known applications of them in various parts of analysis.

The monographic exposition of the theory of Orlicz spaces may be found in the book of Krasnoselskii and Rutickii [3]. For a current review of the theory of Musielak-Orlicz spaces and modular spaces, the reader is referred to the book of Kozlowski [4] and the most recent paper of Khamsi et al. [5], also see [6,7].

As a generalization of the Banach contraction principle, Taleb and Hanebaly [7] presented a fixed point theorem of the Banach type in a modular space where the modular is s-convex, having the Fatou property and satisfying the $\Delta_{2}$-condition as follows.

Theorem 1 ([7]). Let $X_{\rho}$ be a $\rho$-complete modular space. Assume that $\rho$ is an s-convex modular satisfying the $\Delta_{2}$-condition and having the Fatou property. Let $B$ be a $\rho$-closed subset of $X_{\rho}$ and $T: B \rightarrow B$ a mapping such that:

$$
\exists c, k \in \mathbb{R}^{+}: c>\max \{1, k\}, \rho(c(T x-T y)) \leq k^{s} \rho(x-y) \forall x, y \in B .
$$

Then $T$ has a fixed point. 
In this paper, by means of [7], we prove the existence of fixed points for a general class of contractive mappings satisfying Schauder and Petryshyn conditions in s-modular function spaces. We give an application of our result to a nonlinear integral equation in Musielak-Orlicz spaces.

\section{Preliminaries}

We begin by recalling some definitions. Let $X$ be a linear space over $\mathbb{C}$. Then we have the following.

(1) A function $\rho: X \rightarrow[0,+\infty]$ is said to be modular if

(a) $\rho(x)=0$ if and only if $x=0$;

(b) $\rho(\alpha x)=\rho(x)$ for all scalar $\alpha$ with $|\alpha|=1$;

(c) for all $x, y \in X, \rho(\alpha x+\beta y) \leq \rho(x)+\rho(y)$ if $\alpha+\beta=1$ for any $\alpha, \beta \geq 0$;

(2) If (c) is replaced by

(c') $\rho(\alpha x+\beta y) \leq \alpha^{s} \rho(x)+\beta^{s} \rho(y)$ if $\alpha^{s}+\beta^{s}=1$ for any $\alpha, \beta \geq 0$, where, if $0 \leq s<1$, then we say that $\rho$ is an s-convex modular and if $s=1$, then $\rho$ is convex modular;

(3) A modular $\rho$ defines a corresponding modular space, i.e., the vector space $X_{\rho}$ given by

$$
X_{\rho}=\{x \in X: \rho(\lambda x) \rightarrow 0 \text { as } \lambda \rightarrow 0\} .
$$

(4) The modular space $X_{\rho}$ can be equipped with the $F$-norm defined by $|x|_{\rho}=\inf \left\{\alpha>0 ; \rho\left(\frac{x}{\alpha}\right) \leq \alpha\right\}$. If $\rho$ is convex, then the functional

$$
\|x\|_{\rho}=\inf \left\{\alpha>0 ; \rho\left(\frac{x}{\alpha}\right) \leq 1\right\}
$$

is a norm called the Luxemburg norm in $X_{\rho}$ which is equivalent to the $F$-norm $|\cdot| \rho$.

Note that, by taking $\alpha=-1$ in $1(b)$, it follows that $y=\rho(x)=\rho(-x)$, so that a modular is symmetric with respect to the $y$-axis meaning that its graph remains unchanged under reflection about the $y$-axis. It turns out that an $s$-convex modular keeps the same property.

Definition 1. Let $X_{\rho}$ be a modular space.

(a) A sequence $\left\{x_{n}\right\}$ in $X_{\rho}$ is said to be:

(i) $\rho$-convergent to $x$, denoted by $x_{n} \stackrel{\rho}{\rightarrow} x$, if $\rho\left(x_{n}-x\right) \rightarrow 0$ as $n \rightarrow \infty$.

(ii) $\rho$ - Cauchy if $\rho\left(x_{n}-x_{m}\right) \rightarrow 0$ as $n, m \rightarrow \infty$.

(b) $X_{\rho}$ is $\rho$-complete if every $\rho$-Cauchy sequence is $\rho$-convergent.

(c) A subset $B \subseteq X_{\rho}$ is said to be $\rho$-closed if for any sequence $\left\{x_{n}\right\} \subset B$ with $x_{n} \stackrel{\rho}{\rightarrow} x, x \in B$. Also, B is $\rho$-open if $B^{c}$ is $\rho$-closed.

(d) We say that $\partial_{\rho}(B)$ is the bound of a subset $B$ of $X_{\rho}$, whenever

$$
\partial_{\rho}(B)=\bar{B}^{\rho}-\operatorname{int}(B)
$$

where $\bar{B}^{\rho}$ is the closure of $B$ and int $(B)$ is the interior of $B$ in the sense of $\rho$.

(e) $A$ subset $B \subseteq X_{\rho}$ is said to be $\rho$-compact if every sequence in $B$ has a convergent subsequence.

(f) $A$ subset $B \subseteq X_{\rho}$ is called $\rho$-bounded if

$$
\delta_{\rho}(B)=\sup \{\rho(x-y): x, y \in B\}<\infty,
$$

where $\delta_{\rho}(B)$ is called the $\rho$-diameter of $B$.

(g) $\rho$ is said to satisfy the $\Delta_{2}$-condition if $2 x_{n} \stackrel{\rho}{\rightarrow} 0$ whenever $x_{n} \stackrel{\rho}{\rightarrow} 0$. 
(h) We say that $\rho$ has the Fatou property if $\rho(x-y) \leq \liminf \rho\left(x_{n}-y_{n}\right)$ whenever, $x_{n} \stackrel{\rho}{\rightarrow} x$ and $y_{n} \stackrel{\rho}{\rightarrow} y$.

\section{Main Results}

Now, we start our work with the following definitions.

Definition 2. Let $X_{\rho}$ be a modular space and $C \subseteq X_{\rho}$. A mapping $T: C \rightarrow X_{\rho}$ is said to be $\rho$-nonexpansive if $\rho(T x-T y) \leq \rho(x-y)$ for all $x, y \in C$.

Definition 3. A set $C$ of a modular space $X$ is said to be s-convex, where $0<s \leq 1$ if the following condition is satisfied

$$
\alpha^{\frac{1}{s}} x+\beta^{\frac{1}{s}} y \in C \text { whenever } x, y \in C, \quad \alpha+\beta=1 .
$$

We first prove a Schauder type fixed point theorem when the mapping $T$ is $\rho$-nonexpansive.

Theorem 2. Let $\rho$ be an s-convex modular that satisfies the $\Delta_{2}$-condition and Fatou property, $X_{\rho}$ be a $\rho$-complete modular space and $B$ be a nonempty, s-convex, and $\rho$-closed subset of $X_{\rho}$. Assume that $T: B \rightarrow B$ is a $\rho$-nonexpansive operator and $T(B)$ is a subset of $\rho$-compact set of $B$. Then $T$ has a fixed point.

Proof. For every $n \in \mathbb{N}$, define $T_{n}=t_{n}{ }^{\frac{1}{s}} T$, where $\left\{t_{n}\right\} \subseteq(0,1), t_{n} \rightarrow 1$ as $n \rightarrow \infty$. If $s=1$, then $B$ is convex set. Without loss of generality, we assume that $0 \in B$. If $s<1$, then $0 \in B$. Thus for each $n \in \mathbb{N}$, $T_{n}: B \rightarrow B$. There are two cases:

Case-1: Let $s=1$. By putting $c=t_{n} \frac{-1}{2}$ and $k=t_{n}^{\frac{1}{2}}$, we have

$$
\begin{aligned}
\rho\left(c\left(T_{n} x-T_{n} y\right)\right) & =\rho\left(t_{n} \frac{-1}{2}\left(t_{n}(T x-T y)\right)\right) \\
& =\rho\left(t_{n}^{\frac{1}{2}}(T x-T y)\right) \\
& \leq t_{n}^{\frac{1}{2}} \rho(T x-T y) \\
& \leq t_{n}^{\frac{1}{2}} \rho(x-y) \\
& =k \rho(x-y),
\end{aligned}
$$

for all $x, y \in X_{\rho}$.

Case-2: Let $0<s<1$. Set $c=t_{n}^{-1}$ and $k=t_{n}^{\frac{1-s}{s}}$, we obtain

$$
\begin{aligned}
\rho\left(c\left(T_{n} x-T_{n} y\right)\right) & =\rho\left(t_{n}{ }^{-1}\left(t_{n}{ }^{\frac{1}{s}}(T x-T y)\right)\right. \\
& =\rho\left(t_{n}{ }^{\frac{1-s}{s}}(T x-T y)\right) \\
& \leq t_{n}{ }^{1-s} \rho(T x-T y) \\
& \leq t_{n}{ }^{1-s} \rho(x-y) \\
& =k^{s} \rho(x-y),
\end{aligned}
$$

for all $x, y \in X_{\rho}$.

Therefore, all of the assumptions of Theorem 1 hold. Thus for each $n \in \mathbb{N}, T_{n}$ has a fixed point $x_{n} \in B$, that is, $x_{n}=T_{n} x_{n}=t_{n} \frac{1}{s} T x_{n}$. 
Since $T(B)$ lies in a $\rho$-compact subset of $B$, we assume without loss of generally that there exists $u \in B$ such that $\rho\left(T x_{n}-u\right) \rightarrow 0$ as $n \rightarrow \infty$. $\Delta_{2}$-condition follows that $\rho\left(2^{\frac{1}{s}}\left(T x_{n}-u\right)\right) \rightarrow 0$ as $n \rightarrow \infty$. Thus,

$$
\begin{aligned}
\rho\left(x_{n}-T x_{n}\right) & =\rho\left(t_{n}^{\frac{1}{s}} T x_{n}-T x_{n}\right) \\
& =\rho\left(\left(1-t_{n} \frac{1}{s}\right) T x_{n}\right) \leq\left(1-t_{n}{ }^{\frac{1}{s}}\right)^{s} \rho\left(T x_{n}\right) \rightarrow 0 \text { as } n \rightarrow \infty .
\end{aligned}
$$

Again $\Delta_{2}$ - condition implies that $\rho\left(2^{\frac{1}{s}}\left(x_{n}-T x_{n}\right)\right) \rightarrow 0$ as $n \rightarrow \infty$. Hence,

$$
\begin{aligned}
\rho\left(x_{n}-u\right) & =\rho\left(x_{n}-T x_{n}+T x_{n}-u\right) \\
& \leq \frac{1}{2} \rho\left(2^{\frac{1}{s}}\left(x_{n}-T x_{n}\right)\right)+\frac{1}{2} \rho\left(2^{\frac{1}{s}}\left(T x_{n}-u\right)\right) \rightarrow 0 \text { as } n \rightarrow \infty .
\end{aligned}
$$

Since $T$ is $\rho$-nonexpansive,

$$
\rho\left(T x_{n}-T u\right) \leq \rho\left(x_{n}-u\right) \text { as } n \rightarrow \infty
$$

Therefore,

$$
\rho(u-T u) \leq \frac{1}{2} \rho\left(2^{\frac{1}{s}}\left(u-T x_{n}\right)\right)+\frac{1}{2} \rho\left(2^{\frac{1}{s}}\left(T u-T x_{n}\right)\right) \rightarrow 0 \text { as } n \rightarrow \infty .
$$

This implies that $u=T u$.

Theorem 3. Let $\rho$ be an s-convex modular that satisfies the $\Delta_{2}$-condition and Fatou property, $X_{\rho}$ be a $\rho$-complete modular space and $B$ be a nonempty, s-convex, and $\rho$-closed subset of $X_{\rho}$. Assume that $T: B \rightarrow B$ is a $\rho$-nonexpansive and $I$ is an identity operator, and $(I-T)(B)$ is $\rho$-closed. Then $T$ has a fixed point.

Proof. Proceeding as in the proof of Theorem 2, one can prove that for each $n \in \mathbb{N}, T_{n}$ has a fixed point $x_{n}$. Thus

$$
\begin{aligned}
\rho\left(x_{n}-T x_{n}\right) & =\rho\left(t_{n}^{\frac{1}{s}} T x_{n}-T x_{n}\right) \\
& =\rho\left(\left(1-t_{n}{ }^{\frac{1}{s}}\right) T x_{n}\right) \\
& \leq\left(1-t_{n}^{\frac{1}{s}}\right)^{s} \rho\left(T x_{n}\right) \rightarrow 0 \text { as } n \rightarrow \infty .
\end{aligned}
$$

The closedness of $(I-T)(B)$ implies that $0 \in(I-T)(B)$. Therefore, there exists $u \in B$ such that $T u=u$.

The following theorem is a new version of the Petryshyn theorem in s-modular function spaces.

Theorem 4. Let $X_{\rho}$ be a complete modular space and $B$ a $\rho$-bounded, $\rho$-open, s-convex subset of $X_{\rho}$ with $0 \in B$. Assume that $\rho$ is an s-convex modular satisfying the $\Delta_{2}$-condition and Fatou property, $T: \bar{B}^{\rho} \rightarrow X_{\rho}$ is a mapping satisfying (1) and the following condition:

$$
x \neq \lambda T x, \quad \forall x \in \partial_{\rho}(B), \lambda \in(0,1) .
$$

Then Thas a fixed point.

Proof. Consider $A:=\{\lambda \in[0,1]: x=\lambda T x$ for some $x \in B\}$. Notice $A$ is nonempty since $0 \in B$. We will show that $A$ is both open and closed in $[0,1]$ and hence $A=[0,1]$.

Let $\alpha^{\frac{1}{s}}$ be the s-conjugate of $c$, i.e., $\frac{1}{c^{s}}+\frac{1}{\alpha}=1$. We first show that $A$ is closed. To see this let $\left\{\lambda_{n}\right\} \subseteq A$ with $\lambda_{n} \rightarrow \lambda$ as $n \rightarrow \infty$. There exists $\left\{x_{n}\right\} \subseteq B$ with $x_{n}=\lambda_{n} T x_{n}$. Since $0 \in B$ and $B$ is $\rho$-bounded, there exists positive real number $M_{0}$ such that $\rho(x) \leq M_{0}$ for all $x \in B$. Condition (1) follows that for all $x \in B, \rho(T x) \leq \rho(x)+\frac{1}{\alpha} \rho\left(\alpha^{\frac{1}{s}} T 0\right)$. By putting $M=M_{0}+\frac{1}{\alpha} \rho\left(\alpha^{\frac{1}{s}} T 0\right)$, we see that $M$ is an upper bound for the set $\{\rho(T x) ; x \in B\}$. For any $n, m \in \mathbb{N}$, we have 


$$
\begin{aligned}
\rho\left(x_{n}-x_{m}\right) & =\rho\left(\lambda_{n} T x_{n}-\lambda_{m} T x_{m}\right) \\
& =\rho\left(\frac{c \lambda_{n}\left(T x_{n}-T x_{m}\right)}{c}+\frac{\alpha^{\frac{1}{s}}\left(\lambda_{n}-\lambda_{m}\right) T x_{m}}{\alpha^{\frac{1}{s}}}\right) \\
& \leq \frac{1}{c^{s}} \rho\left(c \lambda_{n}\left(T x_{n}-T x_{m}\right)\right)+\frac{1}{\alpha} \rho\left(\alpha^{\frac{1}{s}}\left(\lambda_{n}-\lambda_{m}\right) T x_{m}\right) .
\end{aligned}
$$

For large enough numbers $n, m$, we have $\alpha^{\frac{1}{s}}\left|\lambda_{n}-\lambda_{m}\right|<1$ and so

$$
\rho\left(x_{n}-x_{m}\right) \leq\left(\frac{k}{c}\right)^{s} \rho\left(x_{n}-x_{m}\right)+\left|\lambda_{n}-\lambda_{m}\right|^{s} M
$$

Hence,

$$
\rho\left(x_{n}-x_{m}\right) \leq \frac{M}{\alpha\left(1-\left(\frac{k}{c}\right)^{s}\right)}\left|\lambda_{n}-\lambda_{m}\right|^{s} \rightarrow 0 \text { as } n, m \rightarrow \infty .
$$

Since $X_{\rho}$ is complete, we deduce that there exists $x \in \bar{B}^{\rho}$ with $\rho\left(x_{n}-x\right) \rightarrow 0$ as $n \rightarrow \infty$. It follows from $\Delta_{2}$-condition and $\lambda_{n} \rightarrow \lambda$ that

$$
\begin{aligned}
\rho(x-\lambda T x) & =\rho\left(x-x_{n}+x_{n}-\lambda T x\right) \\
& =\rho\left(\frac{c \lambda_{n}\left(T x_{n}-T x\right)}{c}+\frac{\alpha^{\frac{1}{s}}\left(\lambda_{n}-\lambda\right) T x}{\alpha^{\frac{1}{s}}}+\frac{\alpha^{\frac{1}{s}}\left(x-x_{n}\right)}{\alpha^{\frac{1}{s}}}\right) \\
& \leq\left(\frac{k}{c}\right)^{s} \rho\left(x_{n}-x\right)+\left|\lambda_{n}-\lambda\right|^{s} \rho(T x)+\frac{1}{2 \alpha} \rho\left((2 \alpha)^{\frac{1}{s}}\left(x_{n}-x\right)\right) \rightarrow 0 \text { as } n \rightarrow \infty .
\end{aligned}
$$

Thus $\lambda \in A$ and $A$ is closed in $[0,1]$.

Now, let $\lambda_{0} \in A$. Then there exists $x_{0} \in B$ with $x_{0}=\lambda_{0} T x_{0}$. Choose $\epsilon>0$ such that

$$
\epsilon^{s} \leq \min \left\{\alpha^{-1}, \frac{1}{M}\left(1-\left(\frac{k}{c}\right)^{s}\right) r\right\}
$$

where $r=\inf \left\{\rho\left(x-x_{0}\right) ; \quad x \in \partial_{\rho}(B)\right\}$. If $\lambda \in\left(\lambda_{0}-\epsilon, \lambda_{0}+\epsilon\right)$, then for $x \in{\overline{B\left(x_{0}, r\right)}}^{\rho}$ we have

$$
\begin{aligned}
\rho\left(x_{0}-\lambda T x\right) & =\rho\left(\frac{c \lambda\left(T x_{0}-T x\right)}{c}+\frac{\alpha^{\frac{1}{s}}\left(\lambda_{0}-\lambda\right) T x_{0}}{\alpha^{\frac{1}{s}}}\right) \\
& \leq\left(\frac{k}{c}\right)^{s} \rho\left(x_{0}-x\right)+\left|\lambda_{0}-\lambda\right|^{s} \rho\left(T x_{0}\right) \\
& \leq\left(\frac{k}{c}\right)^{s} r+\epsilon^{s} M \\
& \leq\left(\frac{k}{c}\right)^{s} r+\left(1-\left(\frac{k}{c}\right)^{s}\right) r=r .
\end{aligned}
$$

Therefore $\lambda T:{\overline{B\left(x_{0}, r\right)}}^{\rho} \rightarrow{\overline{B\left(x_{0}, r\right)}}^{\rho}$. It is easy to show that $\lambda T$ satisfies the condition (1) and by applying Theorem 1 , we can deduce that $\lambda T$ has a fixed point. Thus there exists $x \in B$ for which $x=\lambda T x$. This shows that $\lambda \in A$ and hence $A$ is open in $[0,1]$.

\section{Application}

In this section, we give an application of Theorem 4 to the following integral equation:

$$
u(t)=u_{0}+\int_{0}^{t} G(t, r) f(r, u(r)) d r ; t \in I=[0,1]
$$


in a modular space $C=C\left([0,1], L^{p}\right)$, where $0<p \leq 1,0<s \leq p$ and

$$
\begin{aligned}
L^{p} & =L^{p}([0,1]) \\
& =\left\{f: f:[0,1] \rightarrow \mathbb{R} \text { is measurable and } \rho(f)=\int_{0}^{1}|f(t)|^{p} d t<\infty\right\},
\end{aligned}
$$

$u_{0} \in B$ and $B$ is an s-convex, $\rho$-closed, $\rho$-bounded subset of $L^{p}$ with $0 \in B$.

Notice that the $s$-convexity of $\rho$ implies the following lemma.

Lemma 1 ([8]). Let $0<p \leq 1, a \geq 0, b \geq 0$, then $(a+b)^{p} \leq a^{p}+b^{p}$ and $\left|a^{p}-b^{p}\right| \leq|a-b|^{p}$.

We denote by $X=C(I, B)$ the space of all $\rho$-continuous functions from $I$ to $B$, endowed with the modular $\rho_{X}$ defined by $\rho_{X}(u)=\sup _{t \in I} \rho(u(t))$. Using Proposition 2.1 of [7], one can show that $X$ is s-convex, $\rho_{X}$-bounded, $\rho_{X}$-closed of $\rho_{X}$-complete space $C=C\left(I, L^{p}\right)$ and $\rho_{X}$ satisfies the $\Delta_{2}$-condition and Fatou property.

Consider the following assumptions:

(i) $f: I \times B \rightarrow B$ is $\rho$-continuous and satisfies

$$
\exists \gamma>1 \quad \rho(f(t, u)-f(t, v)) \leq \gamma \rho(u-v) ; \quad \forall t \in I \text { and } u, v \in B .
$$

(ii) $G: I \times I \rightarrow \mathbb{R}$ is a measurable mapping such that the map $r \mapsto G(t, r)$ is continuous for almost all $t \in I$, and also $\int_{0}^{1}|G(t, r)| d r<1$ for all $t \in I$.

Theorem 5. Under the conditions (i) and (ii), if for some positive number $\lambda>1$ we have $\lambda \int_{0}^{1}|G(t, r)| d r<$ $\gamma^{\frac{-1}{s}}$, then a mapping $F$ defined on $X$ as

$$
F u(t)=u_{0}+\int_{0}^{t} G(t, r) f(r, u(r)) d r
$$

is a self-adjoint operator which satisfies (1).

Proof. First we show that $F: X \rightarrow X$ is a self-adjoint operator. Suppose $t_{n}, t_{0} \in[0,1]$ and $t_{n} \rightarrow t_{0}$ as $n \rightarrow \infty$. Since $u$ is $\rho$-continuous in $t_{0}$ and condition (i) holds, by $\Delta_{2}$-condition $f$ is $|\cdot|_{\rho}$-continuous where $|.|_{\rho}$ is the F-norm generated by modular $\rho$. Hence, $F u$ is $|.|_{\rho}$-continuous. On the other hand, the topologies generated by $|\cdot|_{\rho}$ and $\rho$ are equivalent, therefore, $F u$ is $\rho$-continuous at $t_{0}$.

Fix $t \in[0,1]$. Let $\lambda>0$ and $T=\left\{t_{0}, t_{1}, \ldots, t_{n}\right\}$ be any subdivision of $[0, t]$. It can be seen that $\sum_{i=1}^{n-1} \lambda\left(t_{i+1}-t_{i}\right) G\left(t, t_{i}\right) u\left(t_{i}\right)$ is $\rho$-convergent to $\int_{0}^{1} \lambda G(t, r) u(r) d r$ when

$$
|T|=\sup \left\{\left|t_{i+1}-t_{i}\right| ; 0 \leq i \leq n-1\right\} \rightarrow 0 \text { as } n \rightarrow \infty .
$$

By the Fatou property, condition (ii) and s-convexity of $\rho$, we have

$$
\begin{aligned}
\rho\left(\int_{0}^{t} \lambda G(t, r) u(r) d r\right) & \leq \liminf \rho\left(\sum_{i=1}^{n-1} \lambda\left(t_{i+1}-t_{i}\right) G\left(t, t_{i}\right) u\left(t_{i}\right)\right) \\
& \leq \lambda^{s}\left(\int_{0}^{1}|G(t, r)| d r\right)^{s} \rho\left(u\left(t_{i}\right)\right) \\
& \leq \lambda^{s}\left(\int_{0}^{1}|G(t, r)| d r\right)^{s} \rho_{X}(u) .
\end{aligned}
$$


This implies that

$$
\begin{aligned}
\rho(\lambda(F u-F v)(t)) & =\rho\left(\int_{0}^{t} \lambda G(t, r)(f(r, u(r))-f(r, v(r))) d r\right) \\
& \leq \lambda^{s}\left(\int_{0}^{1}|G(t, r)| d r\right)^{s} \rho(f(t, u(t))-f(t, v(t))) \\
& \leq \lambda^{s}\left(\int_{0}^{1}|G(t, r)| d r\right)^{s} \gamma \rho(u(t)-v(t)) \\
& =\lambda^{s}\left(\int_{0}^{1}|G(t, r)| d r\right)^{s} \gamma \rho_{X}(u-v) .
\end{aligned}
$$

Therefore,

$$
\rho_{X}(\lambda(F u-F v)) \leq \lambda^{s}\left(\int_{0}^{1}|G(t, r)| d r\right)^{s} \gamma \rho_{X}(u-v) .
$$

By putting $c=\lambda$ and $k=\lambda\left(\int_{0}^{1}|G(t, r)| d r\right) \gamma^{\frac{1}{s}}$, the operator $F$ satisfies the condition (1).

Theorem 6. Under the conditions (i) and (ii), suppose there exists $\rho_{X}$-bounded open $U \subseteq X$ with $0 \in U$ such that if $u$ solves the integral equation

$$
u(t)=k u_{0}+\int_{0}^{t} k G(t, r) f(r, u(r)) d r
$$

for some $k \in(0,1)$, then $u \notin \partial_{\rho_{X}}(U)$. Then (3) has a unique solution in $\bar{U}^{\rho_{X}}$.

Proof. Evidently $F: \bar{U}^{\rho_{X}} \rightarrow X$ satisfies in (1). If we apply Theorem 4 and consider the fact that condition (2) occurs because of (4), we get the required result.

Author Contributions: M.R. contributed in conceptualization, investigation, methodology, validation and writing the original draft; H.B. contributed in conceptualization, investigation, methodology, validation and writing the original draft; O.E. contributed in conceptualization, investigation, methodology, validation and writing the original draft; M.D.l.S. contributed in funding acquisition, methodology, project administration, supervision, validation, visualization and editing. All authors agree and approve the final version of this manuscript.

Funding: The authors thank the Basque Government for its support of this work through Grant IT1207-19.

Acknowledgments: The authors thank the Spanish Government and the European Fund of Regional Development FEDER for Grant RTI2018-094336-B-I00 (MCIU / AEI/FEDER, UE) and the Basque Government for Grant IT1207-19. We would like to express our gratitude to the anonymous referees for their helpful suggestions and corrections.

Conflicts of Interest: The authors declare that they have no competing interests concerning the publication of this article.

\section{References}

1. Nakano, H. Modulared Semi-Ordered Linear Spaces; Tokyo Mathematical Book Series; Maruzen Co. Ltd.: Tokyo, Japan, 1950.

2. Musielak, J.; Orlicz, W. On modular spaces. Stud. Math. 1959, 18, 49-65. [CrossRef]

3. Krasnoselskii, M.A.; Rutickii, Y.B. Convex Functions and Orlicz Spaces; Noordhoff Ltd.: Groningen, The Netherlands, 1961.

4. Kozlowski, W.M. Modular Function Spaces, Monographs and Textbooks in Pure and Applied Mathematics; Marcel Dekker: New York, NY, USA, 1988; Volume 122.

5. Khamsi, M.A.; Kozlowski, W.M. Fixed Point Theory in Modular Function Spaces; Springer: New York, NY, USA, 2015.

6. Khamsi, M.A.; Kozlowski, W.M.; Reich, S. Fixed point theory in modular function spaces. Nonlinear Anal. 1990, 14, 935-953. [CrossRef] 
7. Taleb, A.; Hanebaly, E. A fixed point theorem and its application to integral equations in modular function spaces. Proc. Am. Math. Soc. 1999, 128, 419-426. [CrossRef]

8. Ding, G.G. New Theory in Functional Analysis; Academic Press: Beijing, China, 2007.

(C) 2019 by the authors. Licensee MDPI, Basel, Switzerland. This article is an open access article distributed under the terms and conditions of the Creative Commons Attribution (CC BY) license (http://creativecommons.org/licenses/by/4.0/). 